\title{
Synthesis and Evaluation of Novel Biocompatible Super-paramagnetic Iron Oxide Nanoparticles as Magnetic Anticancer Drug Carrier and Fluorescence Active Label
}

\author{
Andriy Shkilnyy, ${ }^{\dagger}$ Emilie Munnier, ${ }^{\dagger}$ Katel Hervé, ${ }^{\dagger}$ Martin Soucé, ${ }^{\dagger}$ Roland Benoit, ${ }^{\dagger}$ \\ Simone Cohen-Jonathan, ${ }^{\dagger}$ Patrice Limelette, ${ }^{\S}$ Marie-Louise Saboungi, ${ }^{\ddagger}$ Pierre Dubois, ${ }^{\dagger}$ and \\ Igor Chourpa*,†
}

\begin{abstract}
EA 4244, Physicochimie des Matériaux et des Biomolécules, Groupe Nanovecteurs Magnétiques pour la Chimiothérapie, Faculté de Pharmacie, Université F. Rabelais, 31 Avenue Monge, F-37200 Tours, France, and IFR-135 Imagerie Fonctionnelle, F-37200 Tours, France, UMR 6619, Centre de Recherche sur la Matière Divisée, CNRS Université d'Orléans, $1 B$ Rue de la Férollerie, F-45071 Orléans Cedex 2, France, and Laboratoire LEMA, UMR 6157 CNRS/CEA, Université F. Rabelais, Parc de Grandmont, 37200 Tours, France
\end{abstract}

Received: November 25, 2009; Revised Manuscript Received: February 23, 2010

\begin{abstract}
We report a one-pot synthesis protocol for highly efficient and stable covalent binding of both the fluorescent drug doxorubicin (DOX) and the biocompatible polymer poly(ethylene glycol) (PEG) to the surface of superparamagnetic iron oxide nanoparticles (SPIONs). The final aim is to obtain a biocompatible, injectable nanosystem combining anticancer activity (magnetically targeted drug delivery) and nondestructive imaging of the treated cancer cells and tissues by means of fluorescence and magnetic resonance imaging (MRI). Our protocol employs silane and epoxide chemistry, which could also be useful to bind other molecules possessing a primary or secondary amine group, such as drugs, proteins, and fluorescent labels. The suspensions of SPIONs-DOX-PEG (iron concentration of $17 \mathrm{mg} / \mathrm{L}$ ) obtained in this study are stable at physiological $\mathrm{pH}$ values. This stability coupled with the PEG surface neutrality makes these nanoparticles compatible with their application in vivo, via systemic administration. Efficient binding of DOX to the SPIONs surface via the amine group of the sugar moiety of the drug, i.e., outside of the aromatic pharmacophore-fluorophore, preserves the fluorescence activity of DOX. Confocal fluorescence spectral imaging of treated MCF7 cancer cells indicates that, in spite of the accumulation of SPIONs-DOX-PEG in the cytosol, only a minor fraction of the drug reaches the nucleus in $24 \mathrm{~h}$. As a result, no in vitro cytotoxicity against MCF7 cancer cells was detected (the highest iron and drug concentrations were $2.7 \mathrm{mg} / \mathrm{L}$ and $8.1 \mu \mathrm{M}$, respectively). Interestingly, SPIONs-DOX particles noncoated with PEG were cytotoxic. We conclude that cellular enzymes can cleave the amine drug-particle linkage, but the PEG shell hinders the cleavage, possibly by sterical repulsion. Therefore, the developed chemistry is useful for stable coating of SPIONs with polymers and fluorescent labels, while an alternative strategy will be needed for more efficient drug release.
\end{abstract}

\section{Introduction}

Super-paramagnetic iron oxide nanoparticles (SPIONs) with a proper surface tuning are developed as promising new systems for clinical applications such as cancer diagnostics (namely, by magnetic resonance imaging, MRI) and cancer treatment (by magnetically targeted drug delivery and/or hyperthermia). For these kinds of applications, multifunctional SPIONs simultaneously modified with biocompatible polymers, anticancer agents, and fluorescence labels are especially interesting, because they combine the possibility of targeted drug delivery and in vivo imaging of treated tumors by means of MRI and fluorescence.

Size, shape, charge, and especially surface properties of such particles strongly influence their behavior in biological environments. It is widely admitted that the size of injectable nanoparticles (NPs) should not exceed $100 \mathrm{~nm} .{ }^{1,2}$ Unlike larger ferromagnetic iron oxide particles, SPIONs smaller than $20 \mathrm{~nm}$

\footnotetext{
* To whom correspondence should be addressed. Tel.: +33(0)247367162. Fax: +33(0)247367270. E-mail: igor.chourpa@univ-tours.fr.

† Faculté de Pharmacie, Université F. Rabelais and IFR-135 Imagerie Fonctionnelle.

* CNRS Université d'Orléans.

§ UMR 6157 CNRS/CEA, Université F. Rabelais.
}

are super-paramagnetic and no longer show magnetic interactions after removal of the magnetic field. They are thus less prone to aggregate and to induce dangerous thrombosis of blood vessels. Proper modification of the SPION surfaces should (i) provide stability against aggregation (within a reasonable concentration range) at physiological $\mathrm{pH}$ and ionic strength, (ii) reduce capture by the body's immune system, and (iii) provide reactive sites suitable for further binding of drugs and biological ligands.

Once coated by a biocompatible polymer, SPIONs are less likely to aggregate and to be captured by the immune system. ${ }^{1}$ There have been numerous reports on stabilization of SPIONs at physiological $\mathrm{pH}$ by the modification of their surface with biocompatible polymers including poly(amino acid), ${ }^{3}$ poly(lactic acid), ${ }^{4}$ dextran, ${ }^{5}$ poly(ethylene glycol), ${ }^{6,7}$ poly(vinyl alcohol),${ }^{8}$ and poly(ethylene glycol fumarate), ${ }^{9}$ etc. Most of these reports include description of either noncovalent or weak covalent surface modification with polymers. For instance, Thunemann et al. ${ }^{3}$ prepared iron oxide NPs coated by electrostatic adsorption of cationic poly(ethylene imine) followed by anionic poly(ethylene oxide)-block-poly(glutamic acid). The obtained NPs formed suspensions of excellent stability under physiological conditions for a period of more than 6 months and were 
suggested as potential MRI contrast agents. Mornet et al. ${ }^{5}$ functionalized SPIONs for MRI applications using weak covalent binding of dextran via Schiff base chemistry and then PEGylated the dextran layer. The chemical binding was intended to provide better stability than in the case of physical adsorption of the polymer. Zhang et al. ${ }^{6}$ prepared PEGylated SPIONs condensing methoxy-PEG-silane on the iron oxide surface. The authors showed that the uptake of the PEGylated SPIONs by the macrophage cells is lower than for nonmodified ones. This was explained by the fact that the presence of neutral PEG on the surface helps to resist charge-mediated protein adsorption and thus to avoid the recognition by macrophages. Lee et al. ${ }^{8}$ prepared stable suspensions of SPIONs by precipitation in poly(vinyl alcohol) solution. Very recently, Mahmoudi et al. ${ }^{9}$ reported on the synthesis of poly(ethylene glycol fumarate) coated SPIONs. The obtained material is a promising drug delivery system since it possesses good biocompatibility and, due to the cross-linked nature of the polymer shell, less "burst effect" compared to the systems with a non-cross-linked shell. Our group recently reported on a method PEGylation of SPIONs by covalent binding via an amide bond. ${ }^{10}$ In the present paper, we propose epoxide chemistry to bind to the SPIONs surface both PEG-5000 (considered as biocompatible in contrast to the lower molecular weight $\mathrm{PEG}^{10}$ ) and the anticancer agent doxorubicin (DOX).

In spite of its severe cardio- and gastrotoxicity, doxorubicin is still used as an effective anticancer drug. ${ }^{11}$ To diminish or completely remove these side effects, targeted drug delivery systems are under development. Pioneering work of Couvreur et al. reported on decreased DOX side effects after coupling the drug to polymer NPs. ${ }^{12}$ There have also been numerous reports on smart polymer- and SPION-based drug delivery systems including reversible, $\mathrm{pH}$-sensitive, or temperaturesensitive DOX-carrier linkages. ${ }^{13-22}$ For instance, Etrich et al. ${ }^{21}$ have developed polymer-DOX conjugates consisting of HPMA copolymers and DOX coupled via acid-sensitive hydrazone linkages. Inspired by the fact that the tumoral environment has a more acidic $\mathrm{pH}$, the authors showed that release of the drug at $\mathrm{pH} 5$ was at least 10 times higher than at $\mathrm{pH}$ 7.4. Yuan et al. ${ }^{22}$ also prepared iron oxide NPs coupled with DOX via hydrazone bonds and subsequently encapsulated them with a thermosensitive smart polymer, chitosan- $g$-poly( $N$-isopropylacrylamide-co- $N, N$-dimethylacrylamide). The polymer used in this experiment exhibited a lower critical solution temperature (LCST) at $38{ }^{\circ} \mathrm{C}$. The authors showed that drug release was low at temperatures below the LCST compared with temperatures above the LCST. Unfortunately, most of the reported systems do not completely exclude uncontrolled drug release.

With respect to the objective study of nanoparticle uptake by cells, this work presents several advantages over a conventional approach. Classically, the nanoparticles are labeled with high-output fluorescent markers attached to the external particle surface. Unfortunately, in these systems, the fluorescence intensity is not always representative of the nanoparticle uptake, since the marker may be cleaved off and no distinction is made between free and particle-bound marker. In our study, the first advantage is that the fluorescent label is buried under a permeable polymeric layer. This protects the fluorophore from cleavage, while allowing the diffusion of small molecules (demonstrated with dimethylsulfoxide (DMSO)) or ions. At the same time, the fluorescence label does not perturb the polymer surface, which can thus be adjusted to optimize the nanoparticle uptake/distribution. The second advantage is that the nanoparticles are labeled with the anticancer drug DOX and not with just any fluorescent marker. The free drug molecule has sitespecific cellular interactions within different cellular compartments. We can thus distinguish the distribution of free drug from that of nanoparticles, in particular since we analyze in detail the intracellular drug interactions by fluorescence spectral imaging.

In our work we set out to develop a versatile SPIONs-based system which offers stable and efficient surface binding of both the biocompatible polymer and the anticancer drug. A one-pot synthesis protocol was applied to covalently link both the fluorescent drug DOX and PEG polymer to the SPIONs surface employing silane and epoxide chemistry. The study describes physical, chemical, and biological properties of the particles. Due to the polymer coating, the nanoparticles are shown to have excellent colloidal stability and their neutral surface can be expected to increase their half-life in the bloodstream. The drug's native fluorescence permitted to follow both the cellular uptake of the nanoparticles and drug release-interaction within cellular compartments. At the same time, to evaluate the possible use of these particles as a drug delivery system, we also studied their cytotoxicity on human breast cancer cells.

\section{Experimental Procedures}

Nanoparticle Synthesis. Chemicals. All chemicals and reagents were analytical grade and used as received without further purification. Methoxy poly(ethylene glycol) amine $\left(M_{\mathrm{w}} 5000 \mathrm{~g} / \mathrm{mol}\right)$ was obtained from Rapp Polymere $\mathrm{GmbH}$ (Tübingen, Germany). (3-Glycidoxypropyl)trimethoxysilane, doxorubicin hydrochloride, dimethylformamide (DMF), and potassium bromide were received from Sigma-Aldrich. Acetone, nitric acid (65\%), iron(III) nitrate nonahydrate, and ammonium hydroxide were purchased from Carlo Erba Reagenti. Hydrochloric acid (37\%), iron(II) chloride tetrahydrate, and iron(III) chloride were obtained from Acros Organics. Ultrapure water was produced using a Barnstead EASYpure RoDi system (Thermo Fisher Scientific). Dialysis membranes (MWCO $8000 \mathrm{Da}$ ) were purchased from Interchim (Montluçon, France).

Synthesis of Initial SPIONs. The iron oxide NPs were prepared according to a co-precipitation protoco ${ }^{10}$ adapted from the literature. ${ }^{23,5}$ In short, the initial iron oxide NPs precipitated upon addition of ammonium hydroxide to a solution containing iron(II) and iron(III) chlorides. Thereafter, the outer shell of iron oxide NPs was further oxidized to maghemite with iron(III) nitrate at $100{ }^{\circ} \mathrm{C}$. The nanoparticles were peptized with nitric acid, then precipitated, and washed thrice in acetone. Finally, they were re-dispersed in water and stored at $\mathrm{pH} 2.5$ and at 4 ${ }^{\circ} \mathrm{C}$. The concentration of iron was determined using atomic absorption spectroscopy and was found to be $21.8 \mathrm{~g} / \mathrm{L}$. As a control for biological evaluation, a suspension of "bare" SPIONs was prepared in the presence of citrate ions. This treatment ensures their stability at physiological $\mathrm{pH} .{ }^{20}$

SPIONs Surface Modification. A $5 \mathrm{~mL}$ aliquot of an aqueous SPIONs suspension was mixed with $20 \mathrm{~mL}$ of DMF. To get rid of the water, the suspension was concentrated under vacuum to an iron concentration of $30.27 \mathrm{~g} / \mathrm{L}$. The DMF suspension was sonicated for $60 \mathrm{~min}$ prior to the synthesis. Thereafter, 0.638 $\mathrm{g}$ of methoxy poly(ethylene glycol) amine was dissolved in 15 $\mathrm{mL}$ of dry DMF followed by addition of $0.030 \mathrm{~g}$ of (3glycidoxypropyl)trimethoxysilane under a dry nitrogen atmosphere. The system was allowed to react for $4 \mathrm{~h}$ under stirring at room temperature. Afterward, $0.5 \mathrm{~mL}$ of DOX $\left(3.3 \times 10^{-3}\right.$ $\mathrm{M})$ in dry DMF was added to the reaction mixture followed a few minutes later by the addition of $80 \mu \mathrm{L}$ of SPIONs in DMF. The reaction was allowed to proceed for an additional $48 \mathrm{~h}$. 
Thereafter, $9 \mathrm{~mL}$ of a mixture of glycerol and DMF (1:2) was added. The system was concentrated under vacuum to remove the DMF and re-dispersed in $17 \mathrm{~mL}$ of Milli-Q water. The suspension was dialyzed using a cellulose membrane MWCO 8000 for 2 days. The suspension was adjusted to $\mathrm{pH} 7$ to diminish electrostatic stabilization of nonfunctionalized or partially functionalized SPIONs, which were successfully removed by centrifuging at $2350 \mathrm{~g}$ for at least $20 \mathrm{~min}$. The final iron concentration was determind by atomic absorption spectroscopy (AAS) and was found to be $17 \mathrm{mg} / \mathrm{L}$. For reference purposes, suspensions of SPIONs derivatized with PEG alone (SPIONs-PEG) or with DOX alone (SPIONs-DOX) were obtained according to a similar synthesis protocol. For better colloidal stability, the SPIONs-DOX nanoparticles were finally peptized in aqueous solution containing free PEG. All obtained suspensions were stored and kept at $4{ }^{\circ} \mathrm{C}$.

\section{Nanoparticle Characterization}

Physical Properties. Nanoparticle Morphology by Transmission Electron Microscopy. Transmission electron microscopy (TEM) experiments were made on a Philips CM20 transmission electron microscope operated at $200 \mathrm{kV}$. The aqueous suspensions of NPs were deposited on carbon-coated $\mathrm{Cu}$ grids. Excess water was removed by filter paper and drying under ambient air.

Hydrodynamic Diameter and $\zeta$-Potential Measurements. These parameters were measured by means of dynamic light scattering (DLS) at $25^{\circ} \mathrm{C}$. Hydrodynamic diameter (HD) and $\zeta$-potential measurements were conducted on a Malvern Autosizer 4700 and a Malvern NanoZ instrument, both operating with a laser wavelength of $633 \mathrm{~nm}$ and a detection angle of $173^{\circ}$. In a typical experiment $0.5 \mathrm{~mL}$ of the NP suspension was added to $4.5 \mathrm{~mL}$ of water. Samples were not filtered before measurements.

Magnetization Measurements. Magnetization experiments were carried out on a Quantum Design physical property measurement system magnetometer. The magnetization $(M$, $\mathrm{emu} / \mathrm{g}$ ) of freeze-dried modified SPIONs was measured as a function of the magnetic field $(H, O e)$ at $300 \mathrm{~K}$.

Chemical Properties. Iron Content by Atomic Absorption Spectroscopy. AAS measurements were conducted on a SpectrAA 10 Plus atomic absorption spectrometer (Varian). The calibration procedure for the iron absorbance at $248.3 \mathrm{~nm}$ was performed as described before ${ }^{10}$ with a series of Fe solutions with concentrations of 2, 5, 7.4, 10, and $12 \mathrm{mg} / \mathrm{L}$ (prepared from a $2 \mathrm{~g} / \mathrm{L} \mathrm{Fe}$ standard solution). Prior to determination of the Fe concentration in suspensions of SPIONs, the particles were dissolved in concentrated hydrochloric acid (37\%).

Global NP Chemical Composition by FTIR Spectroscopy. Transmission infrared (IR) spectra were recorded using a Fourier transform infrared (FTIR) Spectrometer, model Vector 22 (Bruker). A $5 \mathrm{~mL}$ aliquot of dialyzed suspension was freezedried for $24 \mathrm{~h}$. A $1 \mathrm{mg}$ amount of freeze-dried sample was mixed with $100 \mathrm{mg}$ of potassium bromide and pressed into a pellet using a mechanical press. The spectra were recorded at room temperature from 500 to $4000 \mathrm{~cm}^{-1}$ with a resolution of $4 \mathrm{~cm}^{-1}$.

Elemental Analysis by X-ray Photoelectron Spectrometry. $X$-ray photoelectron spectra were recorded on dry powder using a Thermo Scientific ESCALAB 250 system fitted with a monochromatic Al K $\alpha$ X-ray source operated at $100 \mathrm{~W}$. The size of the X-ray probe was set at $400 \mu \mathrm{m}$, and the samples were cooled with liquid nitrogen. To compensate for surface charging, a low-energy electron gun was used. Wide-scan spectra were acquired for each sample, together with high- resolution $\mathrm{C} 1 \mathrm{~s}, \mathrm{~N} 1 \mathrm{~s}, \mathrm{Si} 2 \mathrm{p}, \mathrm{O} 1 \mathrm{~s}$, and $\mathrm{Fe} 2 \mathrm{p}$ spectra at pass energies of 50 and $20 \mathrm{eV}$. The background was subtracted using a Shirley function calculated from a numerical iterative method. The calibration was carried out at the main C 1s peak at 284.6 $\mathrm{eV}$. Finally, the element concentrations were calculated using the peak intensity and Scofield X-ray photoelectron spectrometry (XPS) cross-sections. ${ }^{24}$

DOX Quantification by UV-vis Spectrophotometry. UV-vis spectra were acquired with an Anthélie advanced spectrophotometer (Secomam). The quantity of DOX released during dialysis was determined from the drug absorbance at $480 \mathrm{~nm}$ $\left(\varepsilon=11500 \mathrm{~cm}^{-1} \mathrm{~mol}^{-1} \mathrm{~L}\right){ }^{20}$ Prior to measurement, the drug was concentrated on a SPE column (Discovery DSC-18 100 $\mathrm{mg}$, Supelco) and eluted with $1 \mathrm{~mL}$ of an $\mathrm{HCl}-$ methanol mixture $(0.1 \mathrm{~mol} / \mathrm{L}, 10 / 90)$.

Biological Properties. Antiproliferative Effect on MCF-7 Cancer Cells. MCF-7 human breast carcinoma cells (American Type Culture Collection, LGC Promochem) were grown in DMEM medium (Fisher Bioblock Scientific) supplemented with $5 \%$ fetal bovine serum (Fisher Bioblock) and $100 \mathrm{UI} / \mathrm{mL}$ penicillin $\mathrm{G}$ and $100 \mu \mathrm{g} / \mathrm{mL}$ streptomycin (Sigma-Aldrich) at $37{ }^{\circ} \mathrm{C}$ in a humidified $5 \% \mathrm{CO}_{2}$ atmosphere.

For cytotoxicity assays, cells were seeded for 1 day in standard 96-well plates (Cellstar, Greiner Bio-One, Courtaboeuf, France) at $5 \times 10^{3}$ cells per well. Then the culture medium was discarded and the cells were treated for $96 \mathrm{~h}$ with $200 \mu \mathrm{L}$ of medium containing different doxorubicin concentrations (16 $\mathrm{nM}$ to $8.1 \mu \mathrm{M}$ ), either as DOX solutions or as suspensions of SPIONs functionalized with DOX alone (SPIONs-DOX) or with both DOX and PEG (SPIONs-DOX-PEG). Control cytotoxicity measurements were also performed in the absence of DOX, for cells incubated with suspensions of citrate-stabilized SPIONs and PEGylated SPIONs and with a PEG solution at the same iron and/or polymer concentration.

Cell viability was determined using a tetrazolium dye (MTT) assay. ${ }^{25}$ The cells were rinsed thrice with phosphate buffered saline (PBS), $\mathrm{pH} 7.4$, and incubated for $3 \mathrm{~h}$ in $100 \mu \mathrm{L}$ of medium containing $0.5 \mathrm{~g} / \mathrm{L}$ MTT (Sigma-Aldrich). Then the medium was replaced by $200 \mu \mathrm{L}$ of DMSO (Sigma-Aldrich) to dissolve the formazan crystals formed by viable cells. Absorbance was measured at $540 \mathrm{~nm}$ using a multiwell plate reader (ELx800 BIO-TEK instruments Inc.). The 50\% inhibitory concentration (IC50) was determined as the drug concentration that resulted in a 50\% reduction in cell viability. All the experiments were performed in triplicate. Data analysis was performed using Origin 5.0 software (Integral Software).

Subcellular Distribution of NPs and Drug Studied by Confocal Fluorescence Spectral Imaging. For the fluorescence imaging study, adherent MCF-7 cells growing in a 24-well plate, on small glass supports $(\phi 8 \mathrm{~mm})$, were incubated with either $1 \mu \mathrm{M}$ DOX solution $(1 \mathrm{~h})$ or with a suspension of SPIONsDOX-PEG containing the drug at ca. $60 \mu \mathrm{M}(3 \mathrm{~h})$. Just before the fluorescence measurements, the cells were rinced twice with fresh medium and placed together with their glass support in a microscopy chamber (Harvard Instruments) thermostatted at 37 $\pm 1{ }^{\circ} \mathrm{C}$, under PBS, pH 7.4, buffer.

Fluorescence spectra were measured with a LabRam laser scanning confocal microspectrometer (Horiba-Jobin-Yvon, Villeneuve d'Ascq, France) equipped with a 300 grooves $/ \mathrm{mm}$ holographic grating and Peltier-cooled CCD detector. The 488 $\mathrm{nm}$ line of an air-cooled argon ion laser (Melles Griot, model 2016) was used to excite the DOX fluorescence (laser power, ca. $10 \mu \mathrm{W}$ on the samples; acquisition time, $0.3 \mathrm{~s}$ per spectrum). Both excitation and spectra collection were performed through 


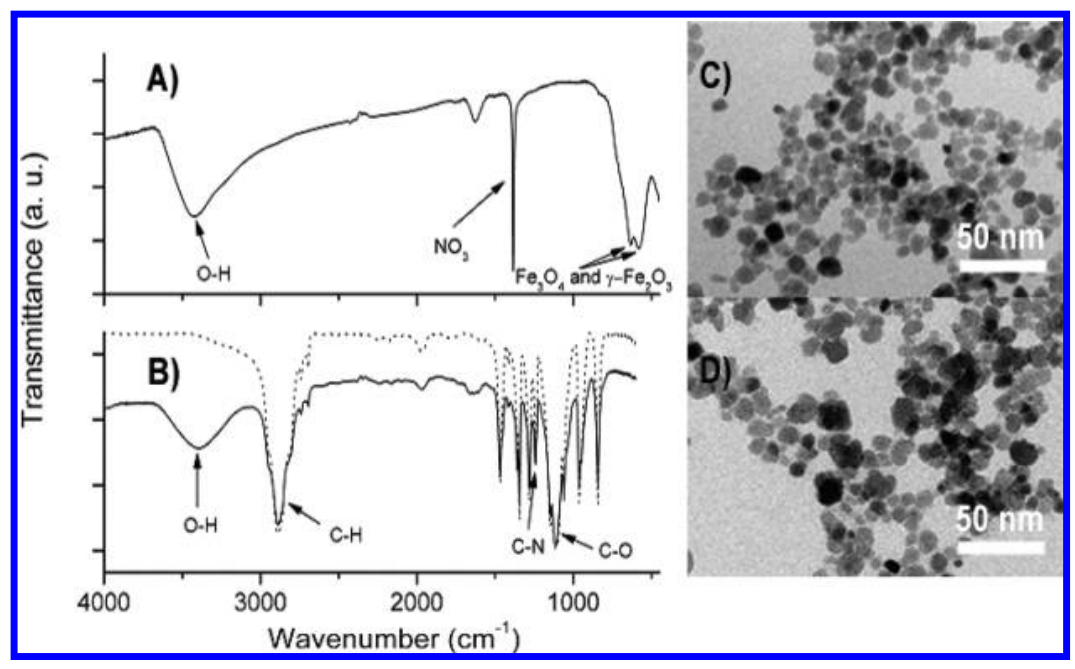

Figure 1. Left: typical FTIR spectra of freeze-dried samples of initial SPIONs (A) and SPIONs-DOX-PEG (B) (the dashed line is a spectrum of initial methoxy poly(ethylene glycol) amine). Right: TEM images of initial SPIONs (C) and SPIONs-DOX-PEG (D).

a $50 \times$ LWD (long working distance) objective (Olympus LMPlanFL, NA 0.5) which allows the cells to be studied through the glass cover of the microscopy chamber described above. Equatorial optical sections of selected cells were scanned with a $0.8 \mu \mathrm{m}$ step that provided maps containing typically 625 spectra $(25 \times 25$ points $)$. Both acquisition and treatment of spectral maps were performed with LabSpec software.

Subcellular drug and particle distribution maps were established via analysis of both the intensity and shape of DOX fluorescence spectra, as described previously. ${ }^{26}$ Briefly, each experimental spectrum was fitted with a sum of reference fluorescence spectra weighted with appropriate coefficients (fitting scores). These scores were used to generate the respective two-dimensional distribution maps over the cell. The results were averaged over 10 cells for each kind of treatment.

\section{Results and Discussion}

Initial SPIONs. The initial suspension of SPIONs was prepared as described previously. ${ }^{10}$ The nanoparticles were precipitated from a basic medium containing iron(II) and iron(III) salts. The material obtained is known to consist of two iron oxide phases, namely, magnetite $\left(\mathrm{Fe}_{3} \mathrm{O}_{4}\right)$ (particle core) and maghemite $\left(\gamma-\mathrm{Fe}_{2} \mathrm{O}_{3}\right)$ (superficial layers). ${ }^{27}$ The treatment of nanoparticles with iron nitrate and nitric acid ensures the enrichment of the superficial maghemite layer and prevents further uncontrollable oxidation.

To confirm the chemical composition of the initial material, we performed FTIR analysis. The FTIR spectrum of the freezedried initial SPIONs (Figure 1A) confirms the presence of the two iron oxide phases maghemite and magnetite. ${ }^{28,29}$ In agreement with literature, the band at $630 \mathrm{~cm}^{-1}$ is assigned to maghemite, while the band at $590 \mathrm{~cm}^{-1}$ corresponds to both magnetite and maghemite vibrations. ${ }^{29}$ The strong narrow band at $1380 \mathrm{~cm}^{-1}$ is due to traces of $\mathrm{NO}_{3}{ }^{-}$anions on the surface of SPIONs. There is also a wide band at $3400 \mathrm{~cm}^{-1}$ attributed to surface $\mathrm{OH}$ groups of SPIONs. These data were in agreement with Raman microspectroscopy analysis described previously ${ }^{27}$ that also indicated that magnetite and maghemite were the two major oxide species, at a relative fraction of ca. 60 and $40 \%$, respectively.

The morphology and size of initial SPIONs were analyzed by means of transmission electron microscopy (Figure 1C) and DLS. The nanoparticles appear to have a uniform roughly spherical morphology, with an average size of ca. $10 \mathrm{~nm}$. This value and the DLS data for the aqueous suspension of SPIONs (average $\mathrm{HD}=73 \pm 7 \mathrm{~nm}$ ) are similar to those reported in the literature. ${ }^{10}$

SPIONs Modified with PEG and/or DOX. The surface modification of nanoparticles was performed according to a protocol developed in our group involving the reaction between amine and epoxide functional groups. The use of this type of chemistry offers sufficient reactivity at ambient temperature. In addition, compared to adsorption, the covalent linkage of the drug to the carrier is more stable and will require a specific release mechanism for drug delivery, for instance, enzymatic cleavage.

The synthesis procedure we used to generate SPIONs modified with both DOX and PEG (SPIONs-DOX-PEG) comprises three stages (Scheme 1): (I) reaction between epoxy silane and amine-substituted poly(ethylene glycol), (II) reaction between epoxy silane and doxorubicin, and (III) condensation of the silanized polymer and drug on the surface of SPIONs. To avoid a lengthy separation process and purification of the intermediates, we have combined all synthetic stages into a onepot synthesis procedure. This method allows easy and efficient preparation of PEG- and DOX-modified SPIONs.

Physical Properties of SPIONs-DOX-PEG. The TEM image of SPIONs-DOX-PEG (Figure 1D) shows more or less spherical shape and nearly the same size as those for initial SPIONs, i.e., an average diameter $\sim 10 \mathrm{~nm}$. This can be explained by the fact that the organic surface layer is not visible under the experimental conditions, since it has not sufficient electron density and/or thickness after vacuum drying.

For the SPIONs-DOX-PEG peptized in aqueous medium at $\mathrm{pH} \mathrm{7,} \mathrm{the} \mathrm{average} \mathrm{diameter} \mathrm{of} \mathrm{the} \mathrm{hydrodynamic} \mathrm{diameter}$ distribution was found to be $96 \pm 15 \mathrm{~nm}$. This value is close to those reported in the literature for SPIONs modified with high molecular weight PEG. ${ }^{7,10}$ The higher value of the HD compared with the initial particles should correspond to the presence of the organic layer on the surface of SPIONs. The DLS data indicated that, in contrast to initial SPIONs, modified SPIONs did not show any sign of aggregation at $\mathrm{pH} 7$, indicating PEGmediated stabilization. The steric nature of this stabilization is supported by the fact that the $\zeta$-potential of these samples was close to zero, i.e., $-0.93 \pm 0.08 \mathrm{mV}$, which is concomitant with the neutral surface of PEG. This is in line with an earlier report of our group. ${ }^{10}$ Hervé et al. reported a PEGylation procedure of SPIONs using covalent binding via an amide 
SCHEME 1: One-Pot Synthesis of PEG- and DOX-Modified SPIONs (SPIONs-DOX-PEG)

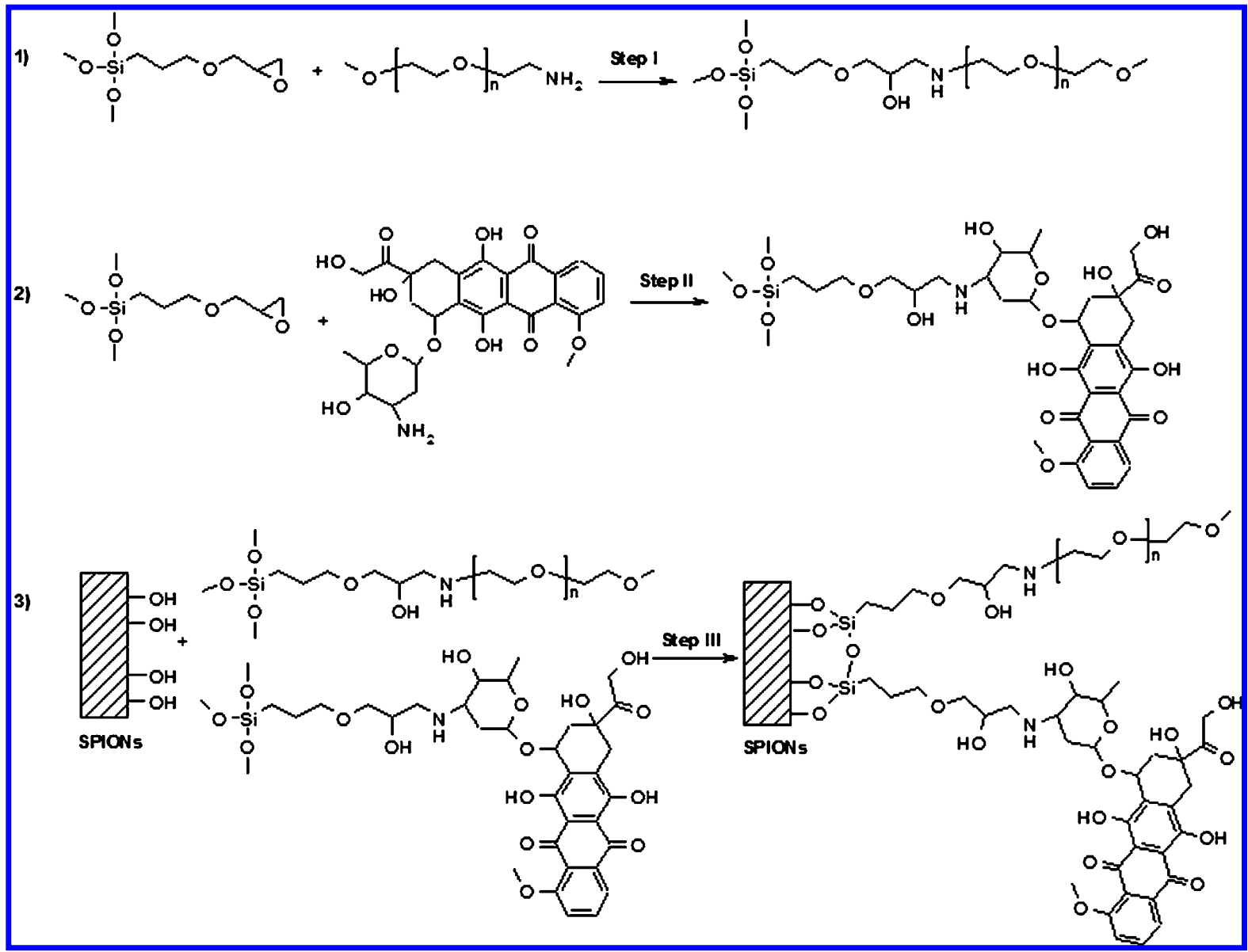

linkage. The authors showed that a neutral molecule such as PEG provides sufficient sterical repulsion to stabilize NPs in suspension within a large range of $\mathrm{pH}(\mathrm{pH} 4-10)$. Furthermore, the fact that the nanoparticle surface is nearly neutral favors the immunological stealthiness required for future biomedical applications in vivo.

The magnetic measurements confirmed that the PEG- and drug-modified samples conserved their superparamagnetic properties. Figure 2 shows the $M$ versus $H$ measurements at room temperature. The saturation magnetization for SPIONs-DOXPEG was equal to ca. $1.12 \mathrm{emu} / \mathrm{g}$ (calculated per total solid mass). This value of magnetization corresponds to the low mass fraction of iron oxide compared to that of the organic component (polymer and drug). If we recalculate the saturation magnetization according to the iron mass fraction (AAS data described

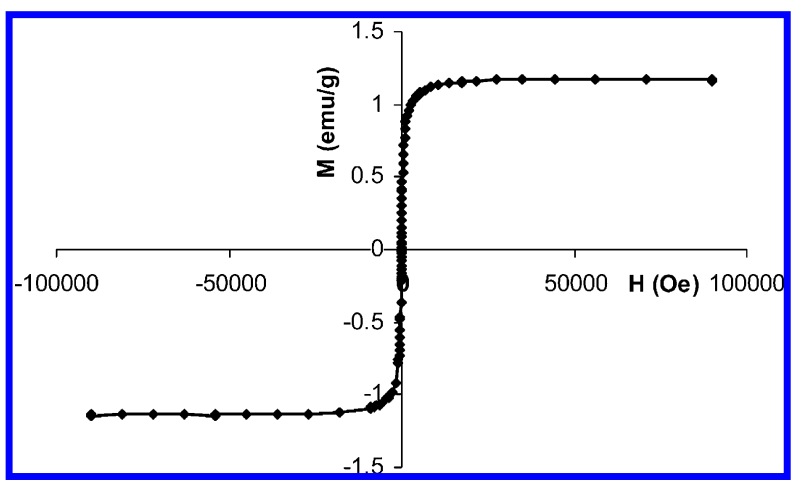

Figure 2. Plot of magnetization $(M)$ versus magnetic field $(H)$ at room temperature for SPIONs modified with PEG and DOX. below), the samples have a magnetization close to $97 \mathrm{emu} /(\mathrm{g}$ of iron). This value correlates well with magnetic data reported for nonmodified and PEGylated SPIONs. ${ }^{10}$ In addition, one can observe the magnetic sensitivity of PEGylated and drug-loaded SPIONs at the microscopic level: the samples move toward a rare earth (neodymium iron boron) magnet of $1.1 \mathrm{~T}$ used in our laboratory for magnetic separation.

Chemical Composition of SPIONs-DOX-PEG. Freeze-dried samples of SPIONs-DOX-PEG contained ca. $1.15 \%(\mathrm{w} / \mathrm{w})$ of iron as determined by AAS. Since the concentration of iron in the sample is lower than the typical sensitivity threshold in IR spectroscopy $(\sim 5 \%(\mathrm{w} / \mathrm{w}))$, the iron oxide bands were no more detectable in the FTIR spectra of modified nanoparticles (Figure 1B). These IR spectra showed the major bands characteristic of PEG at $1113(\mathrm{C}-\mathrm{O}), 1242(\mathrm{C}-\mathrm{N})$, and $2888(\mathrm{CH}) \mathrm{cm}^{-1}$, as well as a band of $\mathrm{OH}$ at $3400 \mathrm{~cm}^{-1}$.

In complement to FTIR that provides the global chemical composition of the NPs, we used XPS to study the chemical composition/bonding on the surface of SPIONs-DOX-PEG. The XPS technique is recognized as a potent tool providing quantitative surface elemental analysis and chemical state information. The typical XPS spectrum of the SPIONs-DOXPEG surface showed mostly carbon, oxygen, and small amounts of nitrogen and silicon (Figure 3). Levels C 1s, O 1s, Si 2p, and $\mathrm{N} 1 \mathrm{~s}$ were deconvoluted into several sets of mixed Gaussian-Lorentzian functions. Si 2 p and N 1s photoemission spectra are shown in the Figure 3 inset. Peak fitting of Si $2 p$ and $\mathrm{N}$ 1s gave respectively the following binding energies: 103.9 \pm 0.2 and $399.9 \pm 0.2 \mathrm{eV}$. These energies correspond to the chemical bonds $\mathrm{Si}-\mathrm{O}_{3}$ and $\mathrm{N}-\mathrm{C}$. Taking into account the depth 


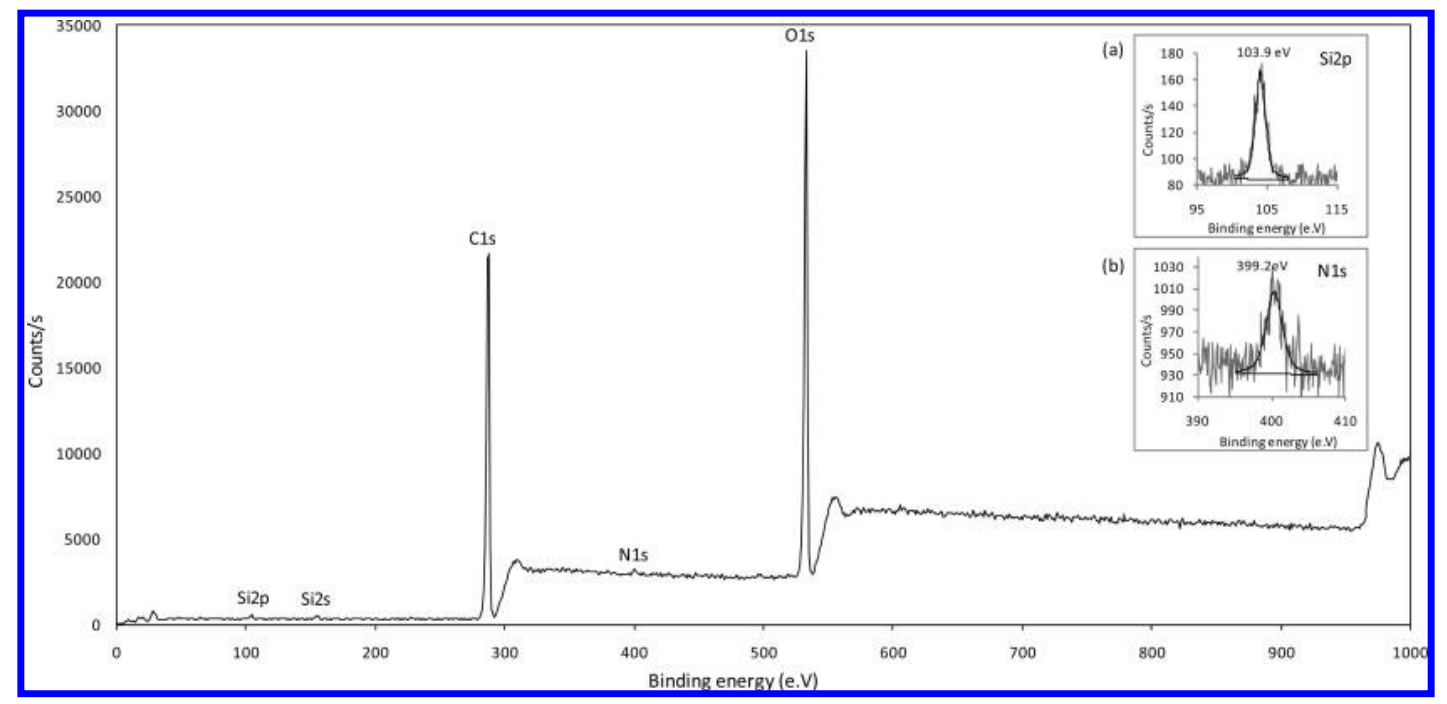

Figure 3. XPS survey spectra of the SPIONs-DOX-PEG sample. Insets: (a) Si 2p XPS spectrum; (b) N 1s XPS spectrum.

TABLE 1: XPS Experimental Data on Atomic Composition within Dried SPIONs-DOX-PEG Samples

\begin{tabular}{lrrrr}
\hline & C 1s & O 1s & Si 2p & N 1s \\
\hline atomic concentration from XPS, \% & 64 & 35 & 0.5 & 0.3 \\
atomic concentration normalized to N & 213 & 117 & 1.7 & 1
\end{tabular}

of analysis estimated according to Cumpson ${ }^{30}$ at $8 \mathrm{~nm}$, iron could not be quantified. Table 1 provides atomic concentrations (at. \%) estimated from the areas of the C 1s, O 1s, Si 2p, and $\mathrm{N}$ 1s photoelectron lines. In the second line of Table 1, atomic concentrations relative to nitrogen are given. In theory, all nitrogen detected originates from either PEG or DOX bound through the silane to the surface. The $\mathrm{Si} / \mathrm{N}$ and $\mathrm{C} / \mathrm{N}$ ratios are, respectively, representative of the stoichiometry of the epoxideamine reaction or the molecule bound (Scheme 1). According to the chemical structure, for the DOX silane alone a $\mathrm{C} / \mathrm{N}$ ratio of 33 is expected; for the PEG silane alone, the value should be close to 232 (PEG MW 5000).

The similarity of the quantitative results and the nature of the chemical bonds observed on Si $2 p$ and N 1s levels by XPS confirm the presence of a layer of DOX-PEG surrounding the SPIONs. The fact that the percentage of silicon is higher than that of nitrogen seems to suggest that not all epoxide has reacted. However, the difference may not be significant given the experimental uncertainty. The experimental $\mathrm{C} / \mathrm{N}$ ratio of ca. 213 obtained from XPS analysis of SPIONs-DOX-PEG nanoparticles confirms that the polymer constitutes the major fraction in the superficial layers. The difference between 213 and 232 should be due to the presence of DOX, but the experimental error of about $10 \%$ does not permit one to quantify the drug. The presence of DOX on these particles can be more easily demonstrated by the fluorescence measurements described below.

The strong fluorescence signal of DOX we recorded for SPIONs-DOX-PEG suspensions confirmed that the drug content was significant and that the attachment through the amine group of the sugar preserves the fluorescence activity of the chromophore from being quenched. Figure 4 shows typical DOX fluorescence spectra of PEG- and DOX-modified SPIONs suspended in PBS buffer, $\mathrm{pH}$ 7.4. Although recognizable, the fluorescence of the drug linked to the surface of PEGylated SPIONs exhibits a modified spectral shape in comparison to the fluorescence of free DOX in PBS buffer, $\mathrm{pH}$ 7.4: the main maximum is shifted from 596 to $601 \mathrm{~nm}$, and the intensity ratio

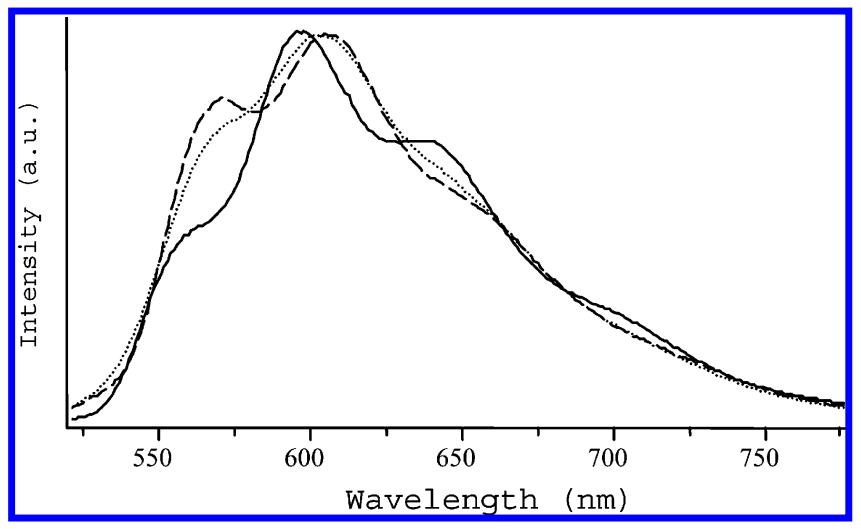

Figure 4. Fluorescence spectra: free DOX in PBS pH 7.4 (solid line); suspension of SPIONs-DOX-PEG in PBS pH 7.4 (dotted line); suspension of SPIONs-DOX-PEG in 80/20 DMSO/water mixture (dashed line).

of the shoulders at ca. 570/635 $\mathrm{nm}$ is increased. This spectral change should correspond to the fact that the drug molecule is immobilized on the SPIONs and buried within a polymeric layer. When SPIONs-DOX-PEG were transferred into a mixture of $80 \%$ DMSO and 20\% water (conditions corresponding to a general solvent effect), this effect is still reinforced. This shows that (i) the environment of DOX in the PEGylated layer has a relatively low polarity and (ii) the PEG coating is permeable to solvent molecules. The latter conclusion is optimistic with respect to the use of PEGylated SPIONs with $\mathrm{pH}$-dependent DOX-SPIONs binding that we proposed recently. ${ }^{20}$

Given the fact that changes in spectral shape are generally accompanied by modified fluorescence quantum yields, it was difficult to use the fluorescence intensity to estimate the DOX concentration within a sample of SPIONs-DOX-PEG. The amount of the drug attached to the surface of nanoparticles was estimated by means of UV-visible spectrophotometry from the mass balance between initially added DOX and the sum of recovered DOX in dialyzates collected for 2 days (see Experimental Procedures). The dialyzates contained less than $1 \%$ of the initially incubated drug. Even if we were to allow for up to $10 \%$ of the drug lost due to adsorption on glassware, this indicates an extremely high efficiency of drug loading and a high stability of the drug linkage in aqueous suspension.

Biological Evaluation of the Obtained Nanoparticles. $D O X$ and Particle Distribution in Living Cells. The fluorescence signal from SPIONs-DOX-PEG allowed us to follow the 


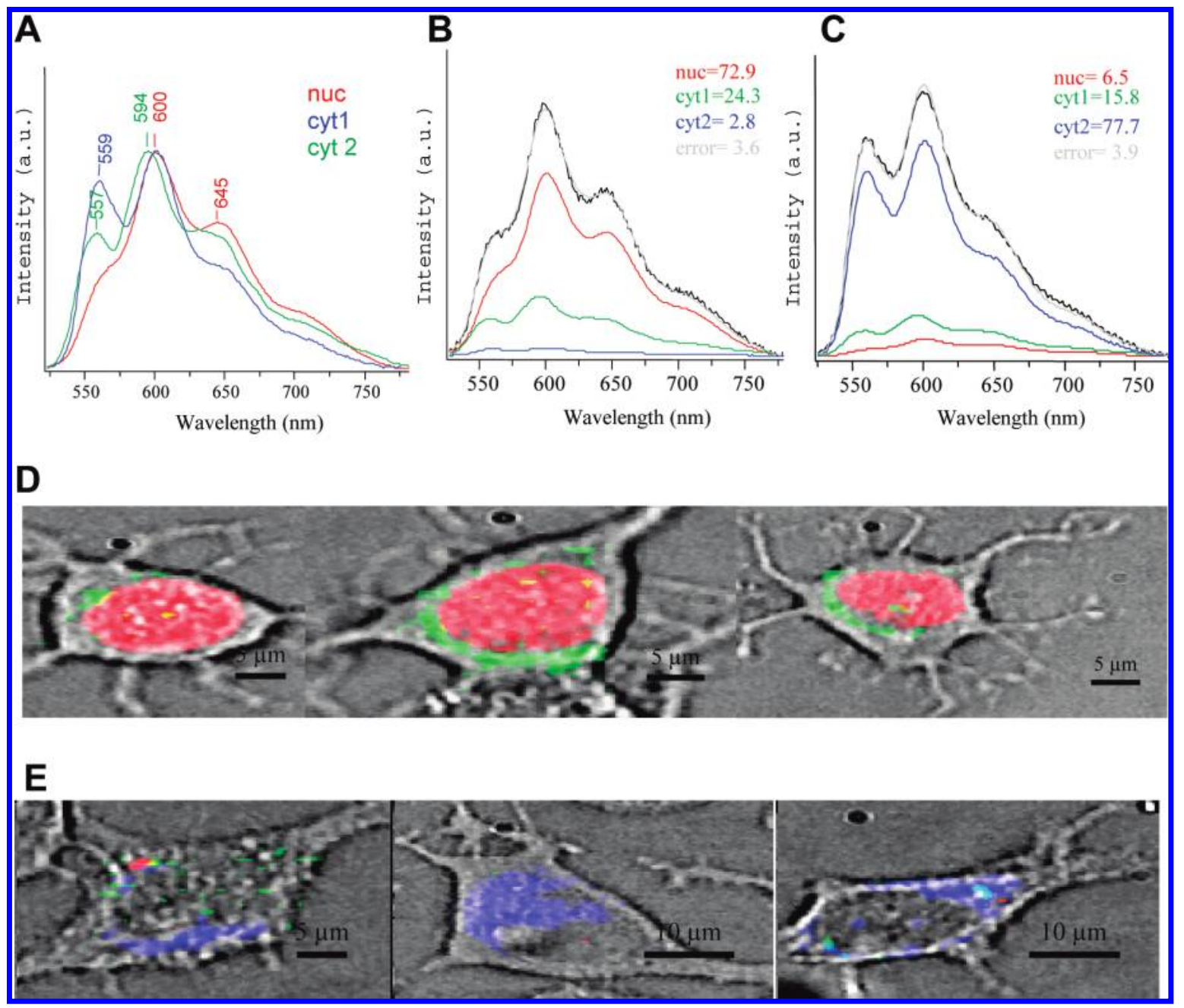

Figure 5. (A) Reference spectra used to fit the intracellular DOX fluorescence corresponding to the drug located in the nucleus (red curve denoted nuc), in two cytosolic environments, respectively, of lower polarity (blue curve denoted cyt1) and of higher polarity (green curve denoted cyt2). Cell-averaged fluorescence from MCF-7 cancer cells incubated with (B) DOX solution or (C) SPIONs-DOX-PEG. Spectral fluorescence maps for MCF-7 cells treated with (D) DOX solution or (E) SPIONs-DOX-PEG.

subcellular distribution of particles by means of confocal spectral imaging (Figure 5). The very important advantage of this method is that the full spectral information is recorded in a spaceresolved manner from each point of the scanned cell.

As expected for PEGylated nanoparticles, SPIONs-DOX-PEG were only slowly internalized by the cells, and we used 60 times higher drug concentrations and at least $3 \mathrm{~h}$ of incubation to detect intracellular fluorescence intensities comparable to those with DOX solution. In $24 \mathrm{~h}$, a moderate increase of the uptake was observed (less than 2-fold, data not shown). To stimulate the uptake by cancer cells, the neutral polymer surface has to be further functionalized with biochemical ligands (folates, antibodies). ${ }^{31}$ This will be the subject of future studies.

Figure 5A shows three characteristic fluorescence spectra we found in different locations of treated cells: one nuclear and two cytosolic ones (denoted as "nuc" and "cyt1" and "cyt2", respectively). These curves were considered as reference spectra. The experimental spectra were fitted with a proportional sum of the three reference spectra taken with contribution coefficients (fitting scores). In this manner, we fitted the spectra averaged over cellular maps, to analyze the relative cellular fraction of the characteristic staining (Figure 5B,C). On the other hand, the fitting scores at each point served us for generating the subcellular drug distribution maps (Figure 5D,E).

The nuclear reference spectrum has a characteristic red shift due to $\pi-\pi$ interaction of DOX intercalated between DNA bases. ${ }^{26}$ As expected for the drug with the preferential nuclear site of action (inhibition of nuclear enzyme topoisomerase II), the cells treated with DOX solution had very intense nuclear fluorescence. It contributed to at least $70-90 \%$ of the overall cellular signal (Figure 5B) and covered all of the nucleus (Figure 5D). In contrast, in the cells treated with SPIONs-DOX-PEG suspension, only weak fluorescence $(5-15 \%$ of the overall signal, Figure 5C) was found in some nuclear points (Figure $5 \mathrm{E})$. In these cells, the dominating staining $(70-85 \%$ of the cellular signal) was cytosolic (Figure 5E) and corresponded to a different spectrum (blue curve denoted cyt1 in Figure 5A,C). This spectrum should correspond to a low-polarity cytosolic environment, because it shows strong changes in shape, similar to those described above for NP in aqueous-organic solvent (DMSO). Interestingly, both types of cell treatment gave rise to another kind of cytosolic fluorescence (green curve named cyt 2 in Figure 5C and green zones on the maps of Figure 5A,B) corresponding to an overall intracellular fraction of $15-25 \%$. According to the spectral shape, this fluorescence may indicate different polarity/interaction than the cyt1 spectrum described above. The fact that, instead of entering the nucleus, the particlecarried drug accumulated in the cytosol (Figure 5E compared to Figure 5D) indicates that the majority of DOX molecules remained bound to SPIONs. Thus, the cyt1 spectrum should rather correspond to internalized SPIONs-DOX-PEG. This is 


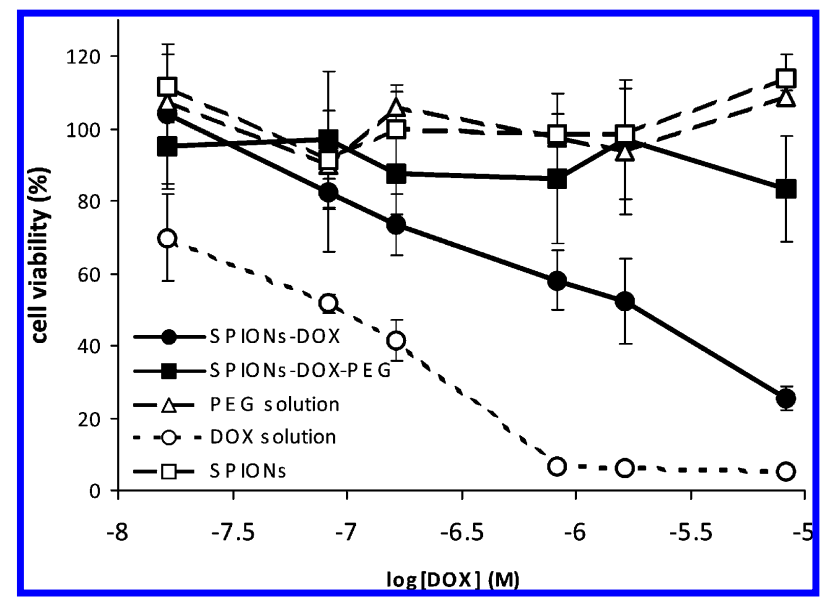

Figure 6. Cytotoxicity evaluation of DOX solution, PEG solution, and suspensions of bare SPIONs (denoted as SPIONs) and of SPIONs functionalized either with DOX (denoted as SPIONs-DOX) or with DOX and PEG (denoted as SPIONs-DOX-PEG).

confirmed by the negligible presence of the cyt 1 model for cells treated with DOX solution (contribution within fitting errors, Figure 5B).

These data indicate that (i) the SPIONs-DOX-PEG are moderately internalized by the cells; (ii) the internalized SPIONs-DOX-PEG appear preferentially accumulated in membrane-rich cytosolic compartments, possibly endosomes; and (iii) a minor fraction (less than 30\%) of DOX is released (and enters the nucleus) with a significant time delay.

To summarize, the chemistry proposed in this work ensures a stable labeling of the magnetic particles with fluorescent molecules carrying amine groups outside of the fluorochrome and therefore could be useful to study the particle distribution in cells, blood, and tissues. The main drawback of a stable bond between DOX and the nanoparticles is that the immobilized DOX may not be able to interact according to its main mechanism as a drug (inhibition of the nuclear enzyme topoisomerase).$^{26}$ If the active molecule is prevented from entering the nucleus, the antitumor effect might be reduced or delayed. This question will be addressed in the following.

Evaluation of Cytotoxicity of the Obtained Nanoparticles. It needs to be pointed out that, in the experimental conditions used here, neither PEG solution nor drug-free nanoparticles, i.e., uncoated (citrate-stabilized) SPIONs or PEG-coated SPIONs provide any detectable cytotoxicity on MCF-7 cells at the working concentrations (Figure 6).

We compared the antiproliferation activity of SPIONs-DOXPEG suspensions with DOX solutions (taken at the same drug concentrations) on MCF-7 cancer cells. Figure 6 shows the related cytotoxicity profiles, i.e., the cell viability after a $96 \mathrm{~h}$ (three cell cycles) treatment versus drug concentration. Compared to DOX solution, no significant cytotoxicity can be seen for SPIONs-DOX-PEG (Figure 6). First of all, the lack of activity can be due to the relatively low uptake of nanoparticles with neutral PEG surface. To check this hypothesis, we plan to evaluate the anticancer activity of SPIONs-DOX-PEG derivated with folates, which should increase particle uptake by cancer cells. ${ }^{31}$

In the present study, we evaluated the role of PEG in terms of a drug-protecting layer, by using SPIONs-DOX particles where only DOX is covalently bound to SPIONs, without bonded PEG (an equivalent concentration of free PEG is added to the solution for better suspension stability). For the cells treated with SPIONs-DOX, the uptake was not significantly increased compared to SPIONs-DOX-PEG, but a considerable cytotoxicity was found (Figure 6). The LC50 values (lethal concentration for $50 \%$ of cells) over $96 \mathrm{~h}$ were determined to be $80 \mathrm{nM}$ and $3.8 \mu \mathrm{M}$ with DOX and SPIONs-DOX, respectively. It was recently shown ${ }^{9}$ that, under certain conditions (namely, significantly higher iron concentrations and cell densities than those used here), uncoated iron oxide nanoparticles were able to impoverish the culture medium and lead to cell toxicity due to a lack of nutrients, especially proteins. Since no significant cytotoxicity was observed with our uncoated SPIONs, we conclude that the cytotoxicity we observed with SPIONs-DOX could be mainly attributed to the drug.

This activity proves that, in the absence of the PEG protective layer, the bonds between DOX and the SPIONs surface can be cleaved by the cells and that at least a part of the DOX released from SPIONs remains active. These results demonstrate that the chemical linkage we propose to bind DOX to the surface of SPIONs is stable in solution but is biodegradable within cancer cells.

\section{Conclusion}

We developed the one-pot synthesis of colloids of SPIONs modified with both PEG polymer and DOX anticancer drug, as multifunctional systems for anticancer drug delivery and cancer imaging. In contrast to most reports that include more timeand material-consuming preparation procedures, the one-pot synthesis described here is advantageous, in particular in view of a potential production on an industrial scale. Due to the PEG coating, the particles form stable colloidal solutions at physiological $\mathrm{pH}$ values. The drug bound to SPIONs via the sugar moiety preserves both the fluorescence and anticancer activity. Nevertheless, the PEG shell seems to reduce the access of cellular enzymes to the drug-particle linkage and thus limits and/or delays the anticancer effect. Although predictable, this result has a fundamental importance for future nanocarrier development, because it is the experimental demonstration of the fact that both chemical and steric considerations are crucial for efficient drug release from functionalized nanoparticles.

In conclusion, the approach proposed here is useful to efficiently bind polymer and fluorescence label to the SPIONs surface. For fluorescence labeling, the two main advantages are (i) the fluorophore is buried in a low-polarity environment under the polymeric layer and is protected from cleavage and (ii) the fluorophore does not interfere with the biocompatibility of the polymer surface. At the same time, the polymer surface remains accessible for further functionalization with drug and biological ligands ${ }^{31}$ favoring the particle binding to membranes of cancer cells. The obtained results show that the very stable and protected linkage of the drug directly to the iron oxide surface appears not favorable for efficient drug release. Future studies will explore the anticancer activity of PEGylated SPIONs with the drug bound to the polymer, closer to the outer NP shell, or with $\mathrm{pH}$-dependent reversible DOX-SPIONs binding, for instance according to the protocol developed in our laboratory. ${ }^{20}$

Acknowledgment. We are grateful to Pierre-Yves Sizaret and Dr. Brigitte Arbeille for TEM images. The authors acknowledge Région Centre for a postdoctoral fellowship for A.S. and for the financial support of the research on functionalized nanoparticles ("NANOMAG" project). We are grateful to Ligue Nationale Contre Cancer (delegation Loir-et-Cher) for the financial support of instrumental developments for confocal spectral imaging of live cells. 


\section{References and Notes}

(1) Gupta, A. K.; Gupta, M. Synthesis and surface engineering of iron oxide nanoparticles for biomedical applications. Biomaterials 2005, 26 (18), 3995-4021.

(2) Pankhurst, Q. A.; Connolly, J.; Jones, S. K.; Dobson, J. Applications of magnetic nanoparticles in biomedicine. J. Phvs. D: Appl. Phvs. 2003, 36, R167-R181.

(3) Thunemann, A. F.; Schutt, D.; Kaufner, L.; Pison, U.; Mohwald, H. Maghemite nanoparticles protectively coated with poly(ethylene imine) and poly(ethylene oxide)-block-poly(glutamic acid). Langmuir 2006, 22 (5), 2351-2357.

(4) Liu, S.; Wei, X.; Chu, M.; Peng, J.; Xu, Y. Synthesis and characterization of iron oxide/polymer composite nanoparticles with pendent functional groups. Colloids Surf. B 2006, 51 (2), 101-106.

(5) Mornet, S.; Portier, J.; Duguet, E. A method for synthesis and functionalization of ultrasmall superparamagnetic covalent carriers based on maghemite and dextran. J. Magn. Magn. Mater. 2005, 293 (2), 127134.

(6) Zhang, Y.; Kohler, N.; Zhang, M. Surface modification of superparamagnetic magnetite nanoparticles and their intracellular uptake. Biomaterials 2002, 23 (7), 1553-1561.

(7) Xie, J.; Xu, C.; Kohler, N.; Hou, Y.; Sun, S. Controlled PEGylation of monodisperse $\mathrm{Fe}_{3} \mathrm{O}_{4}$ nanoparticles for reduced non-specific uptake by macrophage cells. Adv. Mater. 2007, 19, 3163-3166.

(8) Lee, J.; Isobe, T.; Senna, M. Preparation of ultrafine $\mathrm{Fe}_{3} \mathrm{O}_{4}$ particles by precipitation in the presence of PVA at high $\mathrm{pH}$. $\underline{\text { J. Colloid Interface }}$ Sci. 1996, 177 (2), 490-494.

(9) Mahmoudi, M.; Simchi, A.; Imani, M.; Häfeli, U. O. Superparamagnetic iron oxide nanoparticles with rigid cross-linked polyethylene glycol fumarate coating for application in imaging and drug delivery. $\underline{J \text {. Phvs. }}$ Chem. C 2009, 113, 8124-8131.

(10) Hervé, K.; Douziech-Eyrolles, L.; Munnier, E.; Cohen-Jonathan, S.; Soucé, M.; Marchais, H.; Limelette, P.; Warmont, F.; Saboungi, M. L.; Dubois, P.; Chourpa, I. The development of stable aqueous suspensions of PEGylated SPIONs for biomedical applications. Nanotechnology 2008, 19, 465608.

(11) Tan, M. L.; Choong, P. F. M.; Dass, C. R. Cancer, chitosan nanoparticles and catalytic nucleic acids. J. Pharm. Pharmacol. 2009, 61 (1), 3-12.

(12) Couvreur, P.; Kante, B.; Grislain, L.; Roland, M.; Speiser, P. Toxicity of polyalkylcyanoacrylate nanoparticles II: Doxorubicin-loaded nanoparticles. J. Pharm. Sci. 1982, 71 (7), 790-792.

(13) Zhang, J.; Misra, R. D. K. Magnetic drug-targeting carrier encapsulated with thermosensitive smart polymer: Core-shell nanoparticle carrier and drug release response. Acta Biomater. 2007, 3 (6), 838-850.

(14) Lee, E. S.; Na, K.; Bae, Y. H. Super pH-sensitive multifunctional polymeric micelle. Nano Lett. 2005, 52 (2), 325-329.

(15) Sethuraman, V. A.; Lee, M. C.; Bae, Y. H. A biodegradable pHsensitive Micelle system for targeting acidic solid tumors. Pharm. Res. 2008, 25 (3), 657-666.

(16) Ko, J.; Park, K.; Kim, Y. S.; Kim, M. S.; Han, J. K.; Kim, K.; Park, R. W.; Kim, I. S.; Song, H. K.; Lee, D. S.; Kwon, I. C. Tumoral acidic extracellular $\mathrm{pH}$ targeting of $\mathrm{pH}$-responsive MPEG-poly $(\alpha$-amino ester) block copolymer micelles for cancer therapy. $\underline{\text { J. Controlled Release }}$ 2007, 123 (2), 109-115.
(17) Alexiou, C.; Arnold, W.; Klein, R. J.; Parak, F. G.; Hulin, P.; Bergemann, C.; Erhardt, W.; Wagenpfeil, S.; Lübbe, A. S. Locoregional cancer treatment with magnetic drug targeting. Cancer Res. 2000, 60 (23), 6641-6648.

(18) Lübbe, A. S.; Bergemann, C.; Riess, H.; Schriever, F.; Reichardt, P.; Possinger, K.; Matthias, M.; Dörken, B.; Herrmann, F.; Gürtler, R.; Hohenberger, P.; Haas, N.; Sohr, R.; Sander, B.; Lemke, A. J.; Ohlendorf, D.; Huhnt, W.; Huhn, D. Clinical experiences with magnetic drug targeting: A phase I study with $4^{\prime}$-epidoxorubicin in 14 patients with advanced solid tumors. Cancer Res. 1996, 56 (20), 4686-4693.

(19) Park, S. I.; Lim, J. H.; Hwang, Y. H.; Kim, K. H.; Kim, S. M.; Kim, J. H.; Kim, C. G.; Kim, C. O. In vivo and in vitro antitumor activity of doxorubicin-loaded magnetic fluids. Phys. Status Solidi C 2007, 4 (12), $4345-4351$.

(20) Munnier, E.; Cohen-Jonathan, S.; Linassier, C.; Douziech-Eyrolles, L.; Marchais, H.; Soucé, M.; Hervé, K.; Dubois, P.; Chourpa, I. Novel method of doxorubicin-SPION reversible association for magnetic drug targeting. Int. J. Pharm. 2008, 363 (1-2), 170-176.

(21) Etrich, T.; Jelinkova, M.; Rihova, B.; Ulbrich, K. New HPMA copolymers containing doxorubicin bound via $\mathrm{pH}-$ sensitive linkage: Synthesis and preliminary in vitro and in vivo biological properties. J. Controlled Release 2001, 73 (1), 89-102.

(22) Yuan, Q.; Venkatasubramanian, R.; Hein, S.; Misra, R. D. K. A stimulus-responsive magnetic nanoparticle drug carrier: Magnetite encapsulated by chitosan-grafted-copolymer. Acta Biomater. 2008, 4 (4), 10241037.

(23) Massart, R. Preparation of aqueous magnetic liquids in alkaline and acidic media. IEEE Trans. Magn. 1981, 17 (2), 1247-1248.

(24) Scofield, J. H. Hartree-Slater subshell photoionization cross-sections at 1254 and $1487 \mathrm{eV}$. J. Electron Spectrosc. Relat. Phenom. 1976, 8 (2), $129-137$.

(25) Mosmann, T. Rapid colorimetric assay for cellular growth and survival: Application to proliferation and cytotoxicity assays. J. Immunol. Methods 1983, 65 (1-2), 55-63.

(26) Sharonov, S.; Chourpa, I.; Morjani, H.; Nabiev, I.; Manfait, M.; Feofanov, A. Confocal spectral imaging analysis in studies of the spatial distribution of antitumour drugs within living cancer cells. Anal. Chim. Acta 1994, 290 (1-2), 40-47.

(27) Chourpa, I.; Douziech-Eyrolles, L.; Ngaboni-Okassa, L.; Fouquenet, J. F.; Cohen-Jonathan, S.; Soucé, M.; Marchais, H.; Dubois, P. Molecular composition of iron oxide nanoparticles, precursors for magnetic drug targeting, as characterized by confocal Raman microspectroscopy. Analyst 2005, 130 (10), 1395-403.

(28) Tang, J.; Myers, M.; Bosnic, K. A.; Brus, L. E. Magnetite $\mathrm{Fe}_{3} \mathrm{O}_{4}$ Nanocrystals: Spectroscopic observation of aqueous oxidation kinetics. $\underline{J}$. Phys. Chem. B 2003, 107, 7501-7506.

(29) Tartaj, P.; Morales, M. P.; Veintemillas-Verdaguer, S.; GonzalesCarreno, T.; Serna, J. C. The preparation of magnetic nanoparticles for applications in biomedicine. J. Phvs. D: Appl. Phvs. 2003, 36 (13), R182R196.

(30) Cumpson, P. J. Angle-resolved XPS and AES: Depth-resolution limits and a general comparison of properties of depth-profile reconstruction methods. J. Electron Spectrosc. Relat. Phenom. 1995, 73 (1), 25-52.

(31) Byrne, J. D.; Betancourt, T.; Brannon-Peppas, L. Active targeting schemes for nanoparticle systems in cancer therapeutics. Adv. Drug Deliv. Rev. 2008, 60, 1615-1626.

JP9112188 